\title{
Perspectivas sobre contribuciones y estados del bosque nativo de actores sociales vinculados a la producción ganadera en el Espinal entrerriano
}

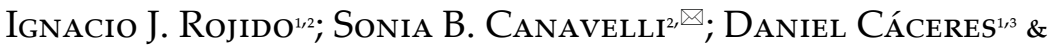 \\ Christopher B. Anderson ${ }^{4,5}$
}

\begin{abstract}
${ }^{1}$ Consejo Nacional de Investigaciones Científicas y Técnicas (CONICET). ${ }^{2}$ Instituto Nacional de Tecnología Agropecuaria (INTA), Estación Experimental Agropecuaria Paraná, Oro Verde, Entre Ríos, Argentina. ${ }^{3}$ Facultad de Ciencias Agropecuarias, Universidad Nacional de Córdoba. Córdoba, Argentina. ${ }^{4}$ Centro Austral de Investigaciones Científicas (CADIC), Consejo Nacional de Investigaciones Científicas y Técnicas (CONICET). Ushuaia, Tierra del Fuego, Argentina. ${ }^{5}$ Instituto de Ciencias Polares, Ambiente y Recursos Naturales (ICPA), Universidad Nacional de Tierra del Fuego (UNTDF). Ushuaia, Tierra del Fuego, Argentina.
\end{abstract}

\begin{abstract}
RESUmen. Los procesos de deforestación y de degradación hacen que el Espinal sea una de las ecorregiones boscosas más amenazadas de la Argentina. En el centro-norte de Entre Ríos, los remanentes de bosques de Espinal nativo son claves para la biodiversidad y para la ganadería integrada al bosque. Por lo tanto, su conservación y uso sostenible requieren incorporar perspectivas de múltiples actores sociales (AS) del sistema productivo. Mediante 21 entrevistas semi-estructuradas con ganaderos familiares y especialistas (investigadores, extensionistas, funcionarios) obtuvimos información sobre sus percepciones acerca de los beneficios que brinda el bosque y su relación con un gradiente de degradación de estados fisonómico-florísticos. Esperábamos que el modo de vida de los ganaderos, vinculado más directamente con el bosque, hiciera que percibieran más beneficios que los especialistas. No obstante, del total de 33 beneficios registrados, 32 fueron reportados por los especialistas y 27 por los ganaderos. Estos beneficios representaron un amplio espectro de contribuciones de la naturaleza para la gente (14 de 18 categorías de IPBES). Ambos AS coincidieron en clasificar la capacidad de provisión de beneficios de los estados del bosque en relación con los modelos de degradación formulados específicamente para la productividad ganadera, atribuyendo pérdidas de beneficios con mayor degradación. No obstante, los ganaderos consideraron que la degradación es provocada en mayor medida por causas naturales, mientras los especialistas responsabilizaron el mal manejo del bosque como factor determinante. A pesar de su degradación, el bosque del Espinal integrado a sistemas ganaderos es percibido como proveedor de múltiples beneficios para la sociedad. Esta información puede aportar a apuntalar la interfaz investigacióngestión del Espinal, incorporando una aproximación constructivista para entender la dimensión humana de los socioecosistemas desde los AS involucrados.
\end{abstract}

[Palabras clave: beneficios de la naturaleza, degradación, evaluación socio-cultural, IPBES, manejo sostenible, Plataforma Intergubernamental Ciencia-Normativa de Biodiversidad y Servicios Ecosistémicos, sistema socioecológico]

\begin{abstract}
Aвstract. Perspectives regarding the contributions and states of native forest by stakeholders linked to livestock production in the Entre Ríos Espinal. Deforestation and degradation processes have made the Espinal one of Argentina's most threatened forested ecoregions. In the central-north part of Entre Ríos Province, forest remnants are important for biodiversity and cattle ranching integrated into the forest. Therefore, the conservation and sustainable use of the Entre Ríos Espinal requires the incorporation of different stakeholders (SH) from this productive system. Using 21 semi-structured interviews with family ranchers and specialists (researchers, extension agents, officials), we collected information about the perceptions of the benefits provided by forests and their relationship with a degradation gradient of physiognomic-floristic states. We expected that the ranchers' lifestyle, linked more directly to the forest, would mean they perceive more benefits, but from the total of 33 recorded benefits, 32 were reported by specialists and 27 by ranchers. These benefits represented a broad spectrum of nature's contributions to people (14 of 18 categories defined by IPBES). While both SH similarly classified the states' capacity to provide benefits in relation to degradation models formulated based on livestock productivity, attributing the loss of benefits to greater degradation, the ranchers considered that the degradation was caused more by natural reasons, while specialists held responsible poor management as the determining driver. Despite its degradation, the Espinal forest integrated into livestock systems is perceived as a provider of multiple benefits for society. These results can contribute to supporting the research-management interface of the Espinal forest, incorporating a constructivist approach to understand the human dimension of socioecosystems from the involved ASs.
\end{abstract}

[Keywords: degradation, Intergovernmental Science-Policy Platform on Biodiversity and Ecosystem Services, IPBES, nature's benefits, sociocultural evaluation, sustainable management, socio-ecological systems]

Editor asociado: Matías Mastrangelo

\ canavelli.sonia@inta.gob.ar
Recibido: 10 de Diciembre de 2019

Aceptado: 3 de Noviembre de 2020 


\section{INTRODUCCIÓN}

Los bosques son grandes reservorios de biodiversidad, ya que albergan más de $75 \%$ de las especies de flora y fauna terrestres a nivel mundial (FAO 2016). Asimismo, desempeñan un rol crucial para múltiples procesos ecosistémicos, como protección del suelo y la captación y transformación de materia y energía en los ciclos biogeoquímicos del agua y el carbono (FAO 2016). A través del marco conceptual de los servicios ecosistémicos (MEA 2005) se demostró que estos ecosistemas realizan contribuciones esenciales para la vida y el desarrollo de las sociedades rurales y urbanas (Krieger 2001; MEA 2005; FAO 2016). En este sentido, se reconoce que los bosques son una parte central del sustento de vida de productores rurales, campesinos e indígenas en todos los continentes, pues mediante éstos satisfacen muchas de sus necesidades de subsistencia, comerciales, culturales, espirituales y recreativas (Vang Rasmussen et al. 2017). Del mismo modo, muchas contribuciones materiales madereras y no madereras (e.g., materiales para construcción y alimentos) (Chiabai et al. 2010), no-materiales (e.g., espacios naturales para experiencias que aportan a la salud física y mental de las personas) (Edwards et al. 2012) y de regulación (e.g., regulaciones climáticas, hidrológicas y edáficas) (Krieger 2001) trascienden el ámbito rural y se vinculan con las sociedades urbanas. A pesar del reconocimiento de la importancia de los bosques por sus beneficios globales, en muchos casos todavía siguen siendo subestimados (Ninan and Kontoleon 2016; Acharya et al. 2019).

La pérdida y la degradación de los bosques por cambios en el uso de la tierra, fragmentación, introducción de especies invasoras, contaminación y sobreexplotación los afecta tanto en sus dimensiones ecológicas como sociales (Ghazoul et al. 2015; IPBES 2018a). En América, los mayores factores de conversión y fragmentación de los ecosistemas son la expansión agrícola industrial y la urbanización (IPBES 2018b). En la Argentina, la mayoría de los ecosistemas boscosos sufrieron una reducción drástica de su superficie (e.g., la Argentina pasó de 32 millones de hectáreas en 1998 a 27 millones en 2015) (Di Pangracio and Cáceres 2020) por un proceso histórico de expansión agropecuaria, intensificado en las últimas dos décadas por la agriculturización de la tierra y la sojización de los cultivos (Schmidt 2018). En la actualidad, la agricultura se conjuga con otras presiones antrópicas sobre los ecosistemas boscosos, como la ganadería comercial intensiva y la urbanización (Nanni et al. 2020). Esta situación de pérdida y degradación de los bosques responde, en gran medida, a una visión utilitarista sobre el ambiente y a la falta de comprensión y valoración de la multifuncionalidad de los territorios (e.g., desconocimiento de la relevancia de los beneficios que brindan los bosques) (Muñoz et al. 2005; Laterra et al. 2011). Como respuesta a la presión social que exigía la protección de los bosques (Schmidt 2018), en 2007 se sancionó la Ley Nacional de Presupuestos Mínimos de Protección de los Bosques Nativos (№ 26331), como política pública para disminuir pérdidas y degradaciones de estos ecosistemas a través de zonificaciones participativas de usos, integraciones de múltiples valores en los criterios de uso y financiamientos de planes de conservación (Guida Johnson and Zuleta 2013). No obstante, todavía no es claro el aporte de esa Ley para disminuir los factores que amenazan los bosques, tanto al nivel nacional como provincial (Aguiar et al. 2018), ya que su aplicación efectiva aún presenta un conjunto de problemas relevantes (e.g., desfinanciamiento, falta de controles, falta de metodologías para valorar los servicios ecosistémicos) (Di Pangracio and Cáceres 2020).

En consonancia con lo visto en otras ecorregiones boscosas de la Argentina, los bosques de Espinal en Entre Ríos sufrieron una pérdida de $\sim 70 \%$ de su superficie (Muñoz et al. 2005; SAyDS 2007; MAyDS 2017). A esta pérdida se suman procesos degradativos que modificaron sus características ecológicas originales (i.e., fisonomía, composición y funciones), incluyendo la intensa utilización histórica de sus recursos madereros (e.g., leña, madera, carbón, postes), el abandono de predios cultivados que genera áreas de renovales, la quema y el sobrepastoreo del ganado (SAyDS 2007; Spahn 2013). En consecuencia, en el centro-norte de Entre Ríos se identifican hoy en día muchos estados fisonómico-florísticos del bosque (Spahn 2013). Los especialistas en manejo ganadero clasifican estos estados en una escala de degradación según características edáficas, fisonómicas y florísticas que inciden sobre su capacidad de producción forrajera (Sabattini et al. 1999; Spahn 2013). De acuerdo con esta clasificación ecológico-productiva, los estados que poseen una alta cobertura arbustiva (>20$25 \%$ ) se consideran degradados (Sione et al. 2006) porque producen menos forraje que 
las áreas más abiertas y, además, dificultan el acceso del ganado, perjudicando la producción ganadera (Wilson and Sabattini 2001). Por lo tanto, los modelos actuales de degradación del Espinal, si bien contemplan aspectos ecológicos, se centran en la pérdida de productividad ganadera.

A pesar desu degradación histórica, el Espinal del centro-norte entrerriano aún tiene un papel importante en los sistemas socioproductivos (Sabattini et al. 1999), ya que la cría extensiva de ganado en bosque es una de las principales actividades económicas de la región (Tasi et al. 2007). Esta ganadería es desarrollada principalmente por productores familiares ( 70\% del total) (Engler et al. 2008). Algunas de estas familias realizan otras actividades vinculadas al bosque, como apicultura, extracción de leña, la producción láctea, la cría de animales de corral, la caza y recolección de frutos silvestres (Casermeiro et al. 2003). Estos productores son actores clave para el manejo y la conservación de este ecosistema, ya que poseen una relación directa y compleja con el bosque: de éste reciben beneficios, aunque también lo disturban (Spahn 2013).

Para gestionar de forma adecuada el bosque, es relevante analizar cómo se relacionan y perciben a este socioecosistema los actores sociales (AS) que lo mantienen como parte integral de sus sistemas socioproductivos (Mastrangelo 2018). Del mismo modo, resulta prioritario indagar sobre las representaciones del bosque de aquellos AS que intervienen en la gobernanza de sus bienes y servicios, a través de generar información científica y de aplicar políticas públicas (Díaz et al. 2011). En el presente trabajo, realizamos entrevistas semiestructuradas para indagar sobre los siguientes aspectos: 1) el reconocimiento de beneficios que provee el bosque, y 2) la percepción de los AS (i.e., los productores ganaderos familiares ['ganaderos'] y los investigadores, extensionistas y funcionarios ['especialistas']) acerca de las características fisonómico-florísticas de los estados del Espinal, de los factores que generan estos estados y de su capacidad para proveer beneficios. Se espera que sus respuestas se vinculen con sus relaciones e intereses con el bosque (e.g., modos de vida) (Quétier et al. 2007). De esta manera, predecimos que los ganaderos, quienes tienen una relación de contacto constante y de múltiples usos directos del bosque, reconocerán más beneficios de este socioecosistema que los especialistas. Además, consideramos que, al pertenecer al ámbito técnico-académico, los especialistas caracterizarán a los estados y su capacidad de proveer beneficios en sintonía con la literatura existente para el área de estudio.

\section{Materiales y Métodos}

\section{Área de estudio}

El centro-norte de Entre Ríos pertenece al Distrito Fitogeográfico del Nandubay del Espinal (Cabrera 1976). Esta ecorregión presenta variaciones fisonómicas, incluyendo bosques semixerofíticos, sabanas y pastizales (SAyDS 2007). El bosque maduro se caracteriza por tener un estrato arbóreo bajo (6-12 m), dominado por ñandubay (Prosopis affinis) y espinillo (Vachellia caven), acompañados por algarrobos (Prosopis spp.), chañar (Geoffroea decorticans), quebracho blanco (Aspidosperma quebracho-blanco) y tala (Celtis ehrenenbergiana), un estrato arbustivo pobre de 2-4 m de altura con una cobertura de hasta 50\% (Cabrera 1976) y un estrato herbáceo continuo con dominancia de pastizales cespitosos (Sabattini et al. 1999).

Este trabajo se desarrolló en los departamentos Feliciano y La Paz. Feliciano (323275 ha) tiene una cobertura de suelo de bosque nativo de $75.6 \%$, del cual $59.7 \%$ corresponde a la categoría I (roja) de conservación prevista por la Ley N²6331, que restringe actividades productivas, y $19.7 \%$ a la categoría II (amarilla), que permite manejo del bosque para actividades productivas (Sabattini et al. 2009a). La Paz (658625 ha) tiene una cobertura del suelo de bosque de $56.3 \%$, del cual $45 \%$ pertenece a categoría roja y $41.3 \%$ a amarilla (Sabattini et al. 2009c). Los dos departamentos concentran entre 15 y $30 \%$ de la producción bovina y ovina de la provincia (Engler et al. 2008), principalmente realizada en campo natural (i.e., bosques, sabanas, pastizales) (Calvi and Rodríguez 2014). En Feliciano, se registra la mayor proporción de superficie destinada a ganadería de la provincia (71\% de la superficie del departamento) (Engler et al. 2008). Las principales actividades agropecuarias de La Paz, en tanto, son la ganadería bovina y la agricultura. Esta última actividad está en expansión (Dupleich and Vicente 2012). En ambos departamentos, $70 \%$ de los productores son familiares, quienes viven en sus explotaciones o en localidades cercanas (Engler et al. 2008; Dupleich and Vicente 2012). Debido al alto número de productores en este sector y su alta dependencia y relación 
con el bosque (Sabattini et al. 1999; Casermeiro et al. 2003), en el presente estudio priorizamos la caracterización de sus perspectivas, ya que anticipamos podrían proveernos una mayor diversidad de valores y relaciones que otros sectores de productores, a la vez que podrían verse más afectados por la degradación del bosque. Desde ya, las perspectivas de este sector de productores constituyen una primera aproximación de valoración sociocultural del Espinal y, por lo tanto, su alcance no abarca la totalidad de esta actividad (e.g., la superficie abarcada por las explotaciones agropecuarias familiares es $\sim 30 \%$ del total en La Paz) (Dupleich and Vicente 2012). En consecuencia, también sería importante abordar las percepciones de los ganaderos no-familiares en futuros estudios, lo cual requiere marcos conceptuales que contemplen el estudio de otros sistemas de gobernanza y toma de decisiones (e.g., economía institucional, administración corporativa).

\section{Recolección de datos}

Adoptamos un enfoque de investigación cualitativo (Sautu et al. 2005) para indagar sobre la perspectiva de los productores ganaderos familiares ('ganaderos'), investigadores, extensionistas y funcionarios ('especialistas') sobre el bosque. Mediante entrevistas semiestructuradas construimos el significado de lo indagado desde el lugar del sujeto de estudio (Mendizábal 2006), y luego vinculamos los resultados con conceptos teóricos y categorías pre-establecidas por el ámbito científico y de la gestión ambiental (Creswell 2014).

El guión de las entrevistas, previamente validado mediante consultas con expertos y pruebas piloto con ganaderos (Rojas Crotte 2011), fue diseñado considerando dos ejes implicados en la evaluación sociocultural de los servicios ecosistémicos (Scholte et al. 2015): 1) percepción de los beneficios que provee el bosque; y 2) percepción de los estados del bosque. Para recabar las percepciones sobre los beneficios que provee el bosque no se utilizaron listas preestablecidas, a fin de no condicionar las respuestas. Para recabar las percepciones sobre los estados, se emplearon fotografías como estímulo visual-cognitivo (LópezSantiago et al. 2014) de seis estados comunes dentro del área de estudio. Se les consultó a los entrevistados sobre tres aspectos de cada fotografía: a) características fisonómicasflorísticas que permiten distinguir al estado, b) factores causantes de estas características fisonómicas-florísticas y c) capacidad de ese estado con respecto a los otros de proveer beneficios (mayor, intermedia, menor).

Los estados, identificados mediante consultas con expertos y revisión bibliográfica (Spahn 2013) fueron seleccionados para que las características fisonómico-florísticas (i.e., altura de los árboles, presencia de renovales, abundancia de especies en los estratos arbustivos y herbáceo) abarquen tres niveles de degradación: a) Poco degradado [1: Bosque semi-maduro con renovales arbóreos y con bajo grado de cobertura arbustiva (3-20\%)]; b) Degradado [2: Bosque semi-maduro sin renovales arbóreos y sin estrato arbustivo; 3: Bosque semi-maduro con renovales, baja cobertura arbustiva $(<20-25 \%)$ y alta cobertura herbácea no-forrajera (Melica macra) (>20-25\%); 4: Bosque semi-maduro con renovales, baja cobertura arbustiva $(<20-25 \%)$ y alta cobertura de subarbusto no-forrajero (Eryngium eburneum) (>20-25\%)]; y c) Muy degradado [5: Bosque renoval con bajo grado de cobertura arbustiva (2-20\%); 6: Bosque renoval con alto grado de cobertura arbustiva (>20-25\%)]. Para mayor descripción, véase Spahn (2013). La relación entre los estados del bosque y el nivel de degradación se estableció sobre la base de estudios que describen, para esta región, atributos ecológicos según su incidencia en la productividad ganadera (Sabattini et al. 1999; Spahn 2013).

\section{Selección de informantes}

Ante la ausencia de un registro oficial, utilizamos una base de datos de extensionistas del INTA con 55 productores ganaderos familiares, caracterizados por: a) residir en el establecimiento o zonas cercanas; b) depender de la ganadería de cría como principal fuente de ingreso; c) aportar al menos la mitad de la mano de obra por la familia; d) obtener el forraje principalmente del campo natural; y e) estar interesados en participar en proyectos de innovación ganadera. La base de datos de especialistas se constituyó identificando a los investigadores $(n=17)$ y extensionistas $(n=3)$ vinculados profesionalmente con el bosque nativo dentro del área de estudio, pertenecientes a la Estación Experimental Agropecuaria Paraná (EEA Paraná-INTA), a la Universidad Nacional de Entre Ríos, a la Universidad Autónoma de Entre Ríos y al Centro Regional de Geomática. Además, se incluyó a funcionarios del Área de Bosque Nativo de la Secretaría de Producción provincial $(\mathrm{n}=2)$. 
La cantidad de entrevistas se definió en base a la redundancia de las respuestas sobre los beneficios (saturación teórica de respuestas) (Soneira 2006) (ver Material Suplementario I), por lo que la muestra es significativa, pero no representativa (Tapella 2012). Entre junio y diciembre del 2017 se realizaron 21 entrevistas. Doce fueron a ganaderos de La Paz $(n=5)$ y Feliciano $(n=7)$, seleccionados mediante un muestreo intencional que abarcó la heterogeneidad de características que podrían incidir en sus relaciones con el bosque (e.g., predios de distintos tamaños, lugar de residencia en el predio o en pueblos, distintas edades) (Mendizábal 2006). Para los especialistas, se realizaron 9 entrevistas (investigadores [n=7], extensionistas $[n=1]$ y funcionarios $[n=1])$.

\section{Interpretación y análisis}

Las entrevistas fueron grabadas y luego transcriptas. El texto se interpretó aplicando un análisis de contenido cualitativo en el que se combinaron procedimientos deductivos (se fijaron anteriormente temas a analizar en base a los objetivos, como ejes de las entrevistas) e inductivos (el material por eje fue codificado utilizando una estrategia de construcción basado en las respuestas) (Mayring 2014).

Beneficios del bosque. Comparamos la percepción de los beneficios del bosque entre los AS y generamos una lista de beneficios para cada uno. Para definir los beneficios se combinó una codificación inductiva inicial basada en lo dicho por los entrevistados, con tipologías sobre servicios ecosistémicos utilizadas en la literatura (Cáceres et al. 2015; Maestre-Andres et al. 2016). Luego estimamos la frecuencia de mención de cada beneficio para cada AS como el número de menciones del beneficio dividido por el número de entrevistados del AS. La frecuencia de mención fue categorizada como baja $(<33 \%)$, media $(33-66 \%)$ y alta $(>66 \%)$. Finalmente, se determinó la relación de los beneficios con las contribuciones de la naturaleza para la gente (CNG) (Díaz et al. 2018).

Estados del bosque y degradación. Comparamos la percepción de los AS sobre las características fisonómicas y florísticas de los estados y los factores causantes de las características, considerando como base nuestra descripción de los estados (sintetizado de Spahn 2013) (véase Recolección de datos). En tanto que la percepción acerca de la capacidad de los estados de proveer beneficios, se comparó mediante el desarrollo de un índice de prominencia (IP) (Sutrop 2001) de cada estado para proveer beneficios, el cual fue contrastado con el gradiente de degradación establecido en base a estudios previos (véase Recolección de datos). El IP se calculó tomando en cuenta el lugar en el ordenamiento hecho por cada entrevistado sobre la capacidad de proveer beneficios (i.e., los estados con capacidad mayor, intermedia o menor), y la frecuencia de mención de esa categorización para cada fotografía entre todos los encuestados:

$$
\mathrm{IP}=\Sigma\left(\mathrm{r}_{\mathrm{i}}^{*} \mathrm{f}\right) / \mathrm{N}
$$

donde el lugar en el ordenamiento de cada fotografía $\left(\mathrm{r}_{\mathrm{i}}\right.$ ) se multiplicó por la frecuencia de mención de cada estado determinado (f), sumando ese producto por cada fotografía y dividiendo porelnúmero total deentrevistados (N). El IP refleja la percepción de cada AS sobre la capacidad para proveer beneficios de cada estado, variando entre 1 (mayor capacidad) y 3 (menor capacidad).

\section{Resultados}

\section{Percepciones sobre beneficios}

Los entrevistados identificaron 33 beneficios que provee el bosque nativo del Espinal del centro-norte de Entre Ríos (Tabla 1). La percepción de ambos actores incluyóbeneficios propios, contribuciones para la sociedad en general y para actividades realizadas por otros. A diferencia de lo esperado, los especialistas reconocieron más beneficios que los ganaderos (32 vs. 27, respectivamente). Sin embargo, los beneficios adicionales descriptos por los especialistas fueron mencionados con baja frecuencia (e.g., materiales para producción de artesanías) y sólo uno con una frecuencia intermedia (estabilidad productiva ganadera). Por su parte, la única contribución del bosque descripta exclusivamente por los ganaderos (regulación del régimen de lluvias, vientos y tormentas) fue mencionada con una frecuencia intermedia (Tabla 1).

Para los especialistas, la mayor cantidad de beneficios reconocidos fueron los materiales (13 de 32), identificando tres más que los ganaderos (Tabla 1). Para ambos AS, los beneficios materiales ligados directamente a la producción ganadera y la leña fueron los mencionados con mayor frecuencia. Para los ganaderos, la mayor cantidad de beneficios identificados fueron los de regulación 
Tabla 1. Beneficios percibidos del bosque nativo en sistemas ganaderos del centro-norte entrerriano. Los beneficios fueron clasificados según el actor social (G: ganaderos y E: especialistas) y las 18 contribuciones de la naturaleza para la gente (CNG), y agrupados en cuatro categorías (Díaz et al. 2018). La frecuencia de mención de cada beneficio está indicada como: A (alta; >66\%), M (media; 66-33\%), y B (baja; <33\%). CNG en letra cursiva no fueron detectados durante las entrevistas.

Table 1. Perceived benefits of the native forest in livestock systems in north-central portion of Entre Ríos. The benefits were classified according to stakeholder (G: ranchers and E: specialists) and to 18 nature's contributions to people (CNG), and grouped into four categories (Díaz et al. 2018). The frequency of mention of each benefit is indicated as: A (high; $>66 \%$ ), M (medium; 66-33\%), and B (low; $<33 \%$ ). CNG in italics were not detected during the interviews.

\begin{tabular}{|c|c|c|c|c|}
\hline \multirow[t]{2}{*}{ Categoría } & \multirow[t]{2}{*}{$\mathrm{CNG}$} & \multirow[t]{2}{*}{ Beneficios identificados en las entrevistas } & \multicolumn{2}{|c|}{$\begin{array}{l}\text { Frecuencia } \\
\text { de } \\
\text { mención }\end{array}$} \\
\hline & & & G & E \\
\hline \multirow[t]{13}{*}{ Material } & \multirow{7}{*}{$\begin{array}{l}\text { Alimento humano y comida } \\
\text { de animales }\end{array}$} & Provisión y protección del clima de pastos forrajeros & A & A \\
\hline & & Otros recursos forrajeros (hojas y frutos de árboles, etc.) & $\mathrm{B}$ & B \\
\hline & & Flores para la producción de miel & M & $\mathrm{M}$ \\
\hline & & Animales silvestres para consumo humano & $\mathrm{B}$ & $\mathrm{M}$ \\
\hline & & Frutos silvestres para consumo humano & B & $\mathrm{M}$ \\
\hline & & Miel de buena calidad & B & $\mathrm{M}$ \\
\hline & & Carne de ganado de buena calidad & & B \\
\hline & \multirow[t]{4}{*}{ Materiales y asistencia } & Postes & A & A \\
\hline & & Madera para edificaciones y muebles & M & B \\
\hline & & Materiales para producción de artesanías. & & B \\
\hline & & Recursos ornamentales (arbustos, etc.) & & B \\
\hline & Energía & Leña & A & A \\
\hline & $\begin{array}{l}\text { Recursos medicinales, } \\
\text { biomédicos y genéticos }\end{array}$ & Plantas medicinales & M & B \\
\hline \multirow[t]{8}{*}{ No-material } & \multirow{3}{*}{$\begin{array}{l}\text { Aprendizaje e inspiración } \\
\text { Apoyo a las identidades }\end{array}$} & Información para aprender & & B \\
\hline & & Sustento del modo de vida (tradiciones y costumbres) & M & A \\
\hline & & Patrimonio cultural de la región & & B \\
\hline & \multirow{5}{*}{$\begin{array}{l}\text { Experiencias físicas y } \\
\text { psicológicas }\end{array}$} & Caza deportiva & $\mathrm{M}$ & B \\
\hline & & Favorece actividades turísticas (ecoturismo; cinegético) & $\mathrm{M}$ & $\mathrm{M}$ \\
\hline & & Contacto con animales y plantas & $\mathrm{M}$ & B \\
\hline & & Valor estético & $\mathrm{M}$ & $\mathrm{M}$ \\
\hline & & Brinda tranquilidad & B & B \\
\hline \multirow[t]{15}{*}{ Regulación } & \multirow[t]{2}{*}{ Regulación del clima } & Regulación de temperatura & B & B \\
\hline & & Secuestro de carbono & B & $\mathrm{M}$ \\
\hline & $\begin{array}{l}\text { Regulación de cantidad, flujo } \\
\text { y periodicidad de agua dulce }\end{array}$ & Regulación del ciclo de agua & B & $\mathrm{M}$ \\
\hline & $\begin{array}{l}\text { Regulación de la calidad de agua } \\
\text { dulce y costera }\end{array}$ & & & \\
\hline & \multirow{2}{*}{$\begin{array}{l}\text { Regulación de riesgos } \\
\text { naturales y eventos extremos }\end{array}$} & Control de inundaciones & B & A \\
\hline & & Regulación de régimen de lluvias, vientos y tormentas & M & \\
\hline & \multirow{3}{*}{$\begin{array}{l}\text { Creación y manutención de } \\
\text { hábitat }\end{array}$} & Refugio para el ganado (bienestar) & A & A \\
\hline & & Resguardo de agroquímicos para la producción de miel & B & $\mathrm{M}$ \\
\hline & & Refugio para biodiversidad & M & A \\
\hline & $\begin{array}{l}\text { Regulación de la calidad de } \\
\text { aire }\end{array}$ & Purificación del aire & A & B \\
\hline & $\begin{array}{l}\text { Regulación de organismos } \\
\text { dañinos para los humanos }\end{array}$ & & & \\
\hline & $\begin{array}{l}\text { Polinización y dispersión de } \\
\text { semillas/propágulos }\end{array}$ & & & \\
\hline & $\begin{array}{l}\text { Regulación de acidificación del } \\
\text { océano }\end{array}$ & & & \\
\hline & \multirow{2}{*}{$\begin{array}{l}\text { Formación, protección y } \\
\text { descontaminación de suelos }\end{array}$} & Aporte de nutrientes para el suelo & B & M \\
\hline & & Prevención de erosión del suelo & B & $\mathrm{M}$ \\
\hline \multirow[t]{2}{*}{ Transversal } & Mantener opciones & Favorece la estabilidad productiva ganadera & & $\mathrm{M}$ \\
\hline & & $\begin{array}{r}\text { Número total de beneficios = } 33 \\
\text { Beneficios compartidos entre G y E } \\
\text { Beneficios específicos por cada actor }\end{array}$ & ${ }_{1}^{27} 26$ & 32 \\
\hline
\end{tabular}


(11 de 27), identificando uno más que los especialistas. De acuerdo con la frecuencia de mención, dentro de esta categoría los ganaderos enfatizaron claramente más que los especialistas el beneficio de purificación del aire $(>66 \%$ y $<33 \%$ para ganaderos y especialistas, respectivamente), mientras que los especialistas destacaron más que los ganaderos el control de inundaciones $(>66 \%$ y $<33 \%$ para especialistas y ganaderos, respectivamente). Además, ambos AS ponderaron un beneficio de regulación ligado a la ganadería (refugio para el ganado). Para ambos AS, la menor cantidad de beneficios reconocidos fueron los no-materiales (6 de 27 y 8 de 32 para ganaderos y especialistas, respectivamente). Dentro de esta categoría, no hubo una diferencia marcada en cuanto a la frecuencia de mención de cada beneficio (Tabla 1).

Los beneficios reconocidos por los AS se enmarcaron en 14 de los 18 tipos de CNG (cuatro materiales, tres no-materiales, siete de regulación y uno transversal [Tabla 1]). La CNG material Alimento humano y comida de animales, fue la que comprendió a una mayor cantidad de beneficios (7 de 33 beneficios), seguida por la CNG no material
Experiencias psicológicas y físicas (5 de 33 beneficios). En particular, la CNG transversal Mantener opciones se vinculó con un beneficio descripto sólo por los especialistas (Favorece la estabilidad productiva ganadera).

\section{Percepción sobre estados y degradación del bosque}

Los especialistas caracterizaron la flora y la fisionomía de cada estado de manera similar a las definiciones de los modelos preestablecidos (Tabla 2), e hicieron hincapié sobre todo en la ausencia de renovales arbóreos (estado 2) y su presencia (estados 3 y 4) (Tabla 2). Simultáneamente, remarcaron que el estado 2, a pesar de que posee un estrato arbóreo maduro, presenta una simplificación fisonómico-florística (e.g., ausencia de renovales arbóreos, ausencia de estrato arbustivo, baja diversidad vegetal) que podría comprometer su capacidad de sobreponerse a disturbios. En cambio, los ganaderos coincidieron de forma parcial con la caracterización basada en los modelos preestablecidos (Tabla 2), y por lo tanto con la de los especialistas, ya que para la mayoría de los estados no reconocieron la presencia o ausencia de renovales. En alusión a los

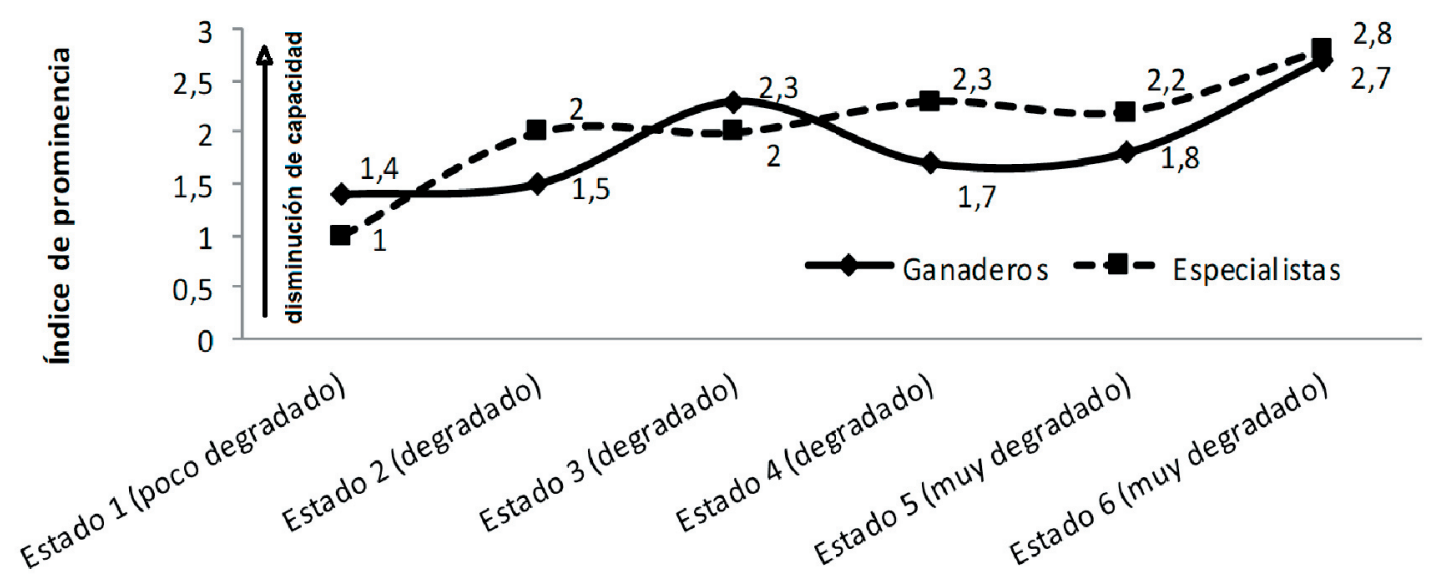

Estados del bosque

Figura 1. Percepción de ganaderos y especialistas sobre capacidad de proveer beneficios de seis estados del bosque nativo en el centro-norte de la Provincia de Entre Ríos, estimada en base a un índice de prominencia (IP) (Sutrop 2001) que combina lugar en el ordenamiento, la frecuencia de mención y el número de entrevistados. La escala va de 1 (mayor capacidad) a 3 (menor capacidad). La relación entre los estados del bosque y el nivel de degradación se estableció en base a estudios realizados en la región (Sabbatini et al. 1999; Spahn 2013), que describen atributos ecológicos centralmente de acuerdo a su incidencia en la productividad ganadera.

Figure 1. Perception of ranchers and specialists on the ability to provide benefits from six native forest ecological states in the north-central portion of Entre Ríos Province, estimated on the basis of a prominence index (IP) (Sutrop 2001), which combines ranking, frequency of mention and number of respondents. The scale ranges from 1 (higher capacity) to 3 (less capacity). The relationship between forest states and the level of degradation was established based on studies carried out in the region (Sabbatini et al. 1999; Spahn 2013), which describe ecological attributes principally according to their effect on livestock productivity. 
Tabla 2. Percepción de ganaderos y especialistas sobre las características fisonómico-florísticas y los factores causantes de seis estados del bosque nativo en el centro-norte de la Provincia de Entre Ríos.

Table 2. Perception of ranchers and specialists on condition and causative factors of six states of the native forest in the north-central portion of Entre Ríos Province.

\begin{tabular}{lll}
\hline $\begin{array}{l}\text { Imágenes y características fisonómicas- } \\
\text { florísticas de los estados (Spahn 2013) }\end{array}$ & $\begin{array}{l}\text { Percepción de los AS sobre las } \\
\text { características de los estados }\end{array}$ & $\begin{array}{l}\text { Percepción de los AS sobre factores } \\
\text { causantes de los estados }\end{array}$ \\
\hline $1-$ & $\begin{array}{l}\text { Ambos actores sociales } \\
\text { *Coincidieron con la descripción } \\
\text { fisonómica-florística }\end{array}$ & $\begin{array}{l}\text { Ganaderos } \\
{ }^{*} \text { Natural }\end{array}$ \\
& $\begin{array}{l}\text { Ganaderos } \\
\text { * Mencionaron que hay un de lena y madera } \\
\text { exceso de renovales }\end{array}$ & $\begin{array}{l}\text { Especialistas } \\
\text { * Carga ganadera adecuada } \\
\text { * Baja carga ganadera }\end{array}$
\end{tabular}

Bosque semi-maduro con renovales y baja cobertura arbustiva (3-20\%)

2-

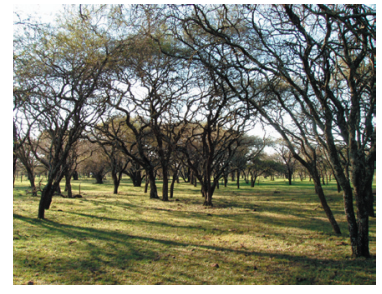

Bosque semi-maduro sin renovales arbóreos y sin estrato arbustivo

3-

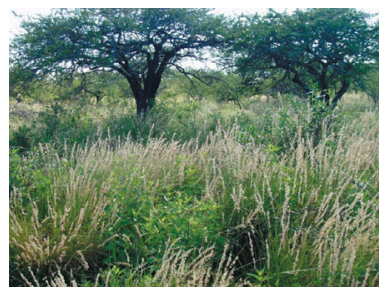

Bosque semi-maduro con renovales, baja cobertura arbustiva $(<20-25 \%)$, alta cobertura herbácea no-forrajera (M. macra) $(>20-25 \%)$

$4-$

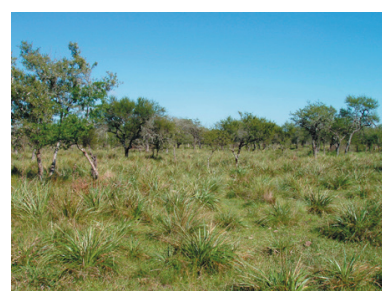

Bosque semi-maduro con renovales, baja cobertura arbustiva ( $<20-25 \%)$ y alta cobertura subarbusto no-forrajera (E. eburneum) $(>20-25 \%)$

5-

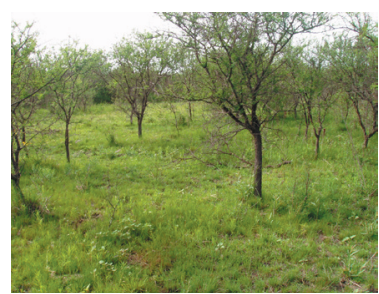

Bosque renoval con baja cobertura arbustiva (2-20\%)
Especialistas

* Coincidieron con la descripción fisonómica-florística

* Enfatizaron la simplificación fisonómica y florística

Ganaderos

* No mencionaron la ausencia de renovales arbóreos

\section{Especialistas}

* Coincidieron con la descripción fisonómica-florística

Ganaderos

* No mencionaron la presencia de renovales arbóreos

Especialistas

* Coincidieron con la descripción fisonómica-florística

\section{Ganaderos}

* No mencionaron la presencia de renovales arbóreos

Ambos actores sociales

* Coincidieron con la descripción fisonómica-florística
Ambos actores sociales

* Sobrepastoreo

* Poda de ramas inferiores de arboles

Ganaderos

* Natural

Especialistas

* Presencia de ovinos

* Control de renovales arbóreos

* Extracción de leña y madera

Ambos actores sociales

* Sobrepastoreo

* Falta de complementariedad bovino/ovino/caprino

* Natural

* Subpastoreo

Ambos actores sociales

* Sobrepastoreo

* Apertura de la cobertura arbórea

Ganaderos

* Natural

Especialistas

* Quema del pastizal

* Desmonte

* Falta de complementariedad

bovino/ovino/caprino

* Ausencia de ganado

Ambos actores sociales

* Desmonte

* Control de arbustos 
Tabla 2. Continuación

Table 2. Continuation

\begin{tabular}{|c|c|c|}
\hline$x+4$ & $\begin{array}{l}\text { Ambos actores sociales } \\
{ }^{*} \text { Coincidieron con la } \\
\text { descripción fisonómica-florística }\end{array}$ & $\begin{array}{l}\text { Ambos actores sociales } \\
\text { * Sobrepastoreo. } \\
\text { * Ausencia de ganado } \\
\text { * Falta de complementariedad } \\
\text { bovino/ovino/caprino } \\
\text { * Falta de control de arbustos } \\
\text { * Desmonte }\end{array}$ \\
\hline $\begin{array}{l}\text { Bosque renoval con alta cobertura arbustiva } \\
(>20-25 \%)\end{array}$ & & $\begin{array}{l}\text { Ganaderos } \\
{ }^{*} \text { Natural } \\
{ }^{*} \text { Subpastoreo } \\
{ }^{*} \text { Falta de poda de árboles }\end{array}$ \\
\hline
\end{tabular}

renovales, los ganaderos consideraron que su presencia (en el estado 1) es algo negativo para su actividad, ya que complejiza el manejo del rodeo.

En cuanto a los factores que causan los estados del bosque, los ganaderos mencionaron entre dos y ocho causas por estado; en algunos casos contrapuestas entre sí (Tabla 2). Por ejemplo, atribuyeron la proliferación de especies no forrajeras (estados 3, 4 y 6) tanto al subpastoreo como al sobrepastoreo. A pesar de la heterogeneidad de causas mencionadas, los ganaderos expresaron reiteradamente causas naturales (e.g., topografía, clima) para la mayoría de los estados $(1,2,3,4$ y 6). De manera similar, los especialistas mencionaron entre dos y siete causas por estado, también algunas contrapuestas entre sí. Por ejemplo, mencionaron al sobrepastoreo y también al subpastoreo como responsables de la proliferación de especies no forrajeras (estados 3 y 4). A diferencia de los ganaderos, los especialistas expresaron pocas causas naturales como determinantes de los estados, y destacaron, en cambio, acciones de manejo para todos los estados. Finalmente, ambos AS coincidieron en que la pérdida de complementariedad de ganadería bovina/ ovina/caprina es uno de los factores que incidió sobre ciertos estados (3 y 6).

Las percepciones de ambos AS sobre la capacidad de proveer beneficios de los estados del bosque coincidieron en gran medida (Figura 1). A su vez, su clasificación fue similar al gradiente de degradación de productividad ganadera, principalmente en sus extremos. El estado 1 (poco degradado) fue identificado por ambos AS como el de mayor capacidad para brindar beneficios; el estado 2 (degradado) fue percibido como el de segunda mayor capacidad para brindar beneficios, y el estado 6 (muy degradado) como aquel con menor capacidad para brindar beneficios (Figura 1). La mayor discrepancia entre la percepción de los AS y el gradiente de degradación fue el estado 5 (muy degradado), percibido por los ganaderos con una capacidad mayor para brindar beneficios que el estado 3 (degradado), y por los especialistas con mayor capacidad que el estado 4 (degradado) (Figura 1).

\section{Discusión}

\section{La relación personas-bosque en sistemas productivos ganaderos}

Los ganaderos y los especialistas percibieron una amplia gama de beneficios del bosque nativo del centro-norte entrerriano. Estos beneficios se relacionaron con todo el espectro de CNG y se vincularon con valores de tipo instrumental (por su valor para satisfacer sus preferencias), intrínseco (por su valor inherente) y relacional (por su valor para relacionarse con la naturaleza y con otras personas) (Chan et al. 2016). No obstante, anticipábamos que los ganaderos percibirían mayor cantidad de beneficios que los especialistas debido a su modo de vida y a su relación más estrecha con el bosque nativo (Quétier et al. 2007), pero encontramos el resultado opuesto. Esta diferencia podría explicarse por un mayor conocimiento de múltiples tipos de ecosistemas boscosos por parte de los especialistas, quienes pueden haber informado beneficios potenciales de los bosques en general, y no sólo de un predio específico (e.g., Información para aprender). Por su parte, las respuestas de los ganaderos podrían vincularse con su dependencia y vivencias cercanas al bosque, ya que, por ejemplo, el único beneficio mencionado exclusivamente por ellos corresponde a la contribución del bosque en disminuir el efecto de los riesgos naturales a los que están expuestos (Regulación de régimen de lluvias, 
vientos y tormentas). La mayor consistencia entre ambos AS en cuanto al reconocimiento de los beneficios brindados por el bosque se observó en la ponderación del rol del bosque como soporte de la ganadería, lo cual reafirma (desde una perspectiva socio-cultural) la importancia del bosque para esta actividad, tal como lo indican estudios productivos y económicos previos (Sabattini et al. 1999; Tasi et al. 2007).

Más allá de las marcadas diferencias socioecológicas en cuanto a las relaciones bosques-sociedades (e.g., tipo de ecosistema boscoso, modo de vida de las personas), en distintas regiones del mundo, diversas poblaciones humanas perciben a los bosques como proveedores de beneficios materiales, no materiales y de regulación relevantes para su bienestar (Tapella 2012; Abram et al 2014). En consonancia, los AS involucrados en nuestro estudio identificaron todo el espectro de beneficios, aunque con mayor proporción de beneficios materiales, seguido por los de regulación $\mathrm{y}$, en menor medida, los nomateriales, en coincidencia con la percepción de diferentes AS sobre los beneficios del bosque de otros ecosistemas boscosos de la Argentina (ecorregión chaqueña) (Cáceres et al. 2015). En consecuencia, los múltiples beneficios del Espinal entrerriano percibidos por los AS aportarían a diferentes aspectos del bienestar de las personas (Díaz et al. 2018) a través de contribuciones de regulación (e.g., regulaciones climáticas e hídricas, hábitat para especies silvestres), contribuciones materiales (e.g. alimentos y energía) y contribuciones no-materiales (e.g., apoyo a las identidades, experiencias físicas y psicológicas).

La relevancia de los ecosistemas boscosos para las personas no solo tiene que ver con los beneficios específicos que brindan, sino que comparativamente con otros ecosistemas naturales, los bosques suelen ser altamente reconocidos por las personas como proveedores de beneficios (ej. Martín-López et al. 2012). Incluso, a estos ecosistemas se los percibe como capaces de brindar mayores proporciones de muchos beneficios materiales, no materiales y de regulación con respecto a otras coberturas de uso del suelo, incluyendo la agricultura a gran escala, que es la cobertura del suelo menos reconocida por su capacidad de brindar beneficios (Brown 2013). Por lo cual, en un contexto provincial de deforestación y agriculturización, y frente a la necesidad de los gestores públicos de identificar y alcanzar sistemas productivos y usos de la tierra que conserven el capital natural como base productiva de la sociedad (Altesor et al. 2011), consideramos relevante el aporte de este estudio para reconocer las perspectivas sociales sobre el bosque del Espinal utilizado para ganadería comercial extensiva.

Las relaciones con los ecosistemas y sus beneficios no son universales, sino que dependen del contexto socio-ecológico de cada región (Díaz et al. 2011). Por esta razón, no deberían ser definidos a priori por los investigadores, sino por los usuarios (Menzel and Teng 2010). El enfoque socioecológico aplicado aquí tiene la fortaleza de incluir la perspectiva de algunos usuarios relevantes para el manejo y la conservación del Espinal del centro-norte entrerriano, permitiendo identificar y definir los beneficios de acuerdo a su perspectiva. A futuro, se necesitarán estudios complementarios que estimen la valoración (e.g., importancia, preferencia) de estos beneficios para los AS incluidos en este estudio y para otros AS (e.g., los productores no-familiares)(Díazetal.2018). Asimismo, sería importante realizar un análisis de los factores que influyen sobre estas valoraciones, como el tipo de actividad productiva desarrollada, la edad, el grado de educación formal alcanzado, etc. (Scholte et al. 2015). De esta forma, sería posible desarrollar un entendimiento más causal entre la valoración sociocultural y los mecanismos que las explican (Maestre-Andrés et al. 2016).

\section{La relación entre la capacidad del bosque de brindar beneficios y el manejo ganadero}

Los AS considerados aquí atribuyeron una mayor capacidad de proveer beneficios a los estados del bosque clasificados a priori como menos degradados, y una menor capacidad a losidentificados como más degradados, a pesar de que los modelos de degradación utilizados de base para definirlos fueron desarrollados sobre todo en función de la productividad ganadera (Sabattini et al. 1999; Spahn 2013). Este resultado indicaría que, para estos AS, la degradación del Espinal no sólo implica modificaciones fisonómico-florísticas que afectan a la producción ganadera, sino también la pérdida o reducción de múltiples beneficios importantes para la sociedad (Ghazoul et al. 2015; IPBES 2018a). En este sentido, el estado poco degradado sería más favorable no sólo para la ganadería, sino también para proveer beneficios vinculados con todo el espectro 
de CNG, constituyendo una meta deseable desde el punto de vista de la producción y la conservación de estos ecosistemas.

A pesar de las coincidencias entre los AS sobre los estados del bosque y sus capacidades de brindar beneficios, el interés particular de cada AS parecería haber condicionado su percepción sobre aspectos específicos de la degradación (Simula 2009). Los especialistas, cuyo interés profesional se vincula con el manejo ganadero o con la conservación biológica, identificaron la pérdida de rasgos estructurales (e.g., ausencia de renovales arbóreos, simplificación fisonómica) como algo negativo, mientras que los ganaderos, cuyo mayor interés es la producción ganadera, en general no se enfocaron en este aspecto o, incluso, lo valoraron positivamente (Díaz et al. 2011), ya que esto favorecería la provisión de pasto y el manejo del rodeo. La regeneración de los ejemplares arbóreos es central para la conservación del bosque del Espinal (Casermeiro and Spahn 2000), a la vez que se vincula de manera directa con múltiples beneficios (e.g., leña; refugio para el ganado). Por esta razón, sería relevante que los gestores y tomadores de decisiones (incluyendo a los productores ganaderos [e.g., propietarios, principales usuarios y decisores del bosque]) reconozcan y consideren las múltiples perspectivas de los AS involucrados a fin de propiciar medidas de manejo y conservación del bosque en esta ecorregión, factibles de ser aplicadas sin generar conflictos estériles entre AS.

El aspecto central en el que discreparon ambos AS tuvo que ver con los tipos de factores (antrópicos vs. naturales) que consideraron como determinantes de cada estado. Mientras los especialistas vincularon las características de los estados primordialmente con acciones de manejo (e.g., intensidad de pastoreo), los ganaderos enfatizaron los factores naturales (e.g., topografía). Si bien la literatura indica que ambos tipos de factores influyen en la fisonomía y la composición del bosque, la heterogeneidad elevada de estados presentes en el Espinal se debe sobre todo a factores antrópicos (Spahn 2013). Esta diferencia de percepción tiene, nuevamente, implicancias para el manejo y la conservación del bosque, ya que para modificar los comportamientos que provocan las tendencias actuales de degradación es prioritario primero que los ganaderos comprendan cómo sus acciones (e.g., intensidad de pastoreo, tala selectiva, desmonte, quema) inciden sobre la degradación del bosque $\mathrm{y}$, en consecuencia, sobre la capacidad de proveer beneficios. $\mathrm{Si}$ bien existen ejemplos exitosos de recuperación de la oferta de beneficios a través de técnicas de restauración de áreas degradadas, en la mayoría de los casos no se alcanzan los valores de las zonas conservadas (Benayas et al. 2009; Balvanera 2009). Por esta razón, es prioritario promocionar manejos del Espinal que eviten los procesos degradativos.

La gestión y la planificación ambiental depende, en gran medida, de valorar la multifuncionalidad de los ecosistemas (Laterra et al. 2011), de identificar los factores que inciden sobre los comportamientos humanos vinculados a los ecosistemas (Mastrangelo 2018) y de aplicar efectivamente las normativas. Los resultados de este trabajo contribuyen a poner un foco en la dimensión humana de este sistema socioproductivo, inserto en una ecorregión prioritaria para la conservación. Consideramos que este estudio ontribuye con conocimientos sobre los beneficios de los bosques en un país con escasos estudios de este tipo, recabando información sobre todo el espectro de beneficios de los bosques en tierras privadas (Acharya et al. 2019). Con esta aproximación socioecológica esperamos colaborar a apuntalar la interfaz investigación-gestión del Espinal, haciendo explícitas las perspectivas sobre el bosque del Espinal de algunos de los AS más relevantes por la injerencia en su manejo y conservación. También esperamos ayudar a evaluar procesos entrelazados de cambios ecológicos y sociales, a incluir estas perspectivas en la toma de decisiones para la readecuación de las normativas vigentes, y a desarrollar propuestas tecnológicas específicas vinculadas al manejo ganadero en bosques nativos (Aguiar et al. 2018).

Agradecimientos. Agradecemos a los entrevistados y la asistencia técnica de los agentes de extensión S. Boffa, J. Fonseca y M. Pereira. IR fue apoyado por una Beca Doctoral del CONICET y dos proyectos de la Rufford Foundation. La investigación se desarrolló en vinculación con el proyecto PNNAT 1128053 (INTA) y el proyecto FTG-RF-15461-RG (FONTAGRO). 


\section{REFERENCIAS}

Abram, N. K., E. Meijaard, M. Ancrenaz, R. K. Runting, J. A. Wells, D. Gaveau, A. Pellier, and K. Mengersen. 2014. Spatially explicit perceptions of ecosystem services and land cover change in forested regions of Borneo. Ecosystem Services 7:116-127. https://doi.org/10.1016/j.ecoser.2013.11.004.

Acharya, R. P., T. Maraseni, and G. Cockfield. 2019. Global trend of forest ecosystem services valuation. An analysis of publications. Ecosystem Services 39. https://doi.org/10.1016/j.ecoser.2019.100979.

Aguiar, S., M. E. Mastrangelo, M. A. García Collazo, G. H. Camba Sans, C. E. Mosso, L. Ciuffoli, M. Schmidt, M. Vallejos, L. Langbehn, M. Brassiolo, D. M. Cáceres, G. Merlinsky, J. M. Paruelo, L. Seghezzo, L. Staiano, M. Texeira, J. N. Volante, and S. R. Verón. 2018. ¿Cuál es la situación de la Ley de Bosques en la Región Chaqueña a diez años de su sanción? Revisar su pasado para discutir su futuro. Ecología Austral 28:400-417. https://doi.org/10.25260/EA.18.28.2.0.677.

Altesor, A., M. P. Barral, G. Booman, L. Carreño, E. Cristeche, J. P. Isacch, N. Maceira, and N. Pérez 2011. Servicios Ecosistémicos: un marco conceptual en construcción. Aspectos conceptuales y operativos. En P. Laterra, E. G. Jobbágy y J. M. Paruelo (eds.). 2011. Valoración de servicios ecosistémicos: conceptos, herramientas y aplicaciones para el ordenamiento territorial. Buenos Aires: INTA.

Balvanera, P. 2012. Los servicios ecosistémicos que ofrecen los bosques tropicales. Ecosistemas 21(1-2):136-147.

Benayas, J. M. R., A. C. Newton, A. Díaz, and J. M. Bullock. 2009. Enhancement of Biodiversity and Ecosystem Services by Ecological Restoration: A Meta-Analysis. Science 325:1121-1124. https://doi.org/10.1126/science.1172460.

Brown, G. 2013. The relationship between social values for ecosystem services and global land cover: An empirical analysis. Ecosystem Services. https://doi.org/10.1016/j.ecoser.2013.06.004.

Cabrera, A. L. 1976. Enciclopedia Argentina de Agricultura y Jardinería. 2ª ed. Buenos Aires, Editorial ACME. 2(1). Pp. 85.

Cáceres, D. M., E. Tapella, F. Quétier, and S. Díaz. 2015. The social value of biodiversity and ecosystem services from the perspectives of different social actors. Ecology and Society 20(1):62. https://doi.org/10.5751/ES-07297-200162.

Calvi, M., and M. Rodríguez. 2014. Sustentabilidad económica de sistemas ganaderos representativos y mejorados en la zona ganadera homogénea de la región mesopotámica argentina. RIA 40(3):303-311.

Casermeiro, J., and E. Spahn. 2000. Evaluación y guía de condición del bosque nativo entrerriano. Multequina 9:147153.

Casermeiro, J., E. Spahn, D. Bendersky, P. Schlund, M. Revora, and B. Chiapino. 2003. Diagnóstico del estado y uso de los recursos naturales de pequeños productores del centro norte de Entre Ríos. Revista Científica Agropecuaria 7(2):29-35.

Chan, K. M. A., P. Balvanera, K. Benessaiah, M. Chapman, S. Díaz, E. Gómez-Baggethun, R. Gould, N. Hannahs, K. Jax, S. Klain, G. W. Luck, B. Martín-López, B. Muraca, B. Norton, K. Ott, U. Pascual, T. Satterfield, M. Tadaki, J. Taggart, and N. Turner. 2016. Why protect nature? Rethinking values and the environment. PNAS 113:1462-1465. https://doi.org/10.1073/pnas.1525002113.

Chiabai, A., C. Travisi, A. Markandya, H. Ding, and P. Nunes. 2010. Economic Assessment of Forest Ecosystem Services Losses: Cost of Policy Inaction. BC3 Working paper series. Basque Country. https://doi.org/10.1007/s10640-011-94786.

Creswell, J. 2014. Research Design: Qualitative, Quantitative and Mixed Methods Approaches (4th ed.). London: Sage Publications Ltd.

Díaz, S., F. Quétier, D. M. Cáceres, S. Trainor, N. Pérez Harguindeguy, M. S. Bret-Harte, B. Finegan, M. Peña-Claros, and L. Poorter. 2011. Linking functional diversity and social actor strategies in a framework for interdisciplinary analysis of nature's benefits to society. Proceedings of the National Academy of Sciences 108(2):895-902. https: //doi.org/10.1073/pnas.1017993108.

Díaz, S., U. Pascual, M. Stenseke, B. Martín-López, R. T. Watson, Z. Molnár, R. Hill, K. M. A. Chan, I. A. Baste, K. A. Brauman, S. Polasky, A. Church, M. Lonsdale, A. Larigauderie, P. W. Leadley, A. P. E. van Oudenhoven, F. van der Plaat, M. Schröter, S. Lavorel, Y. Aumeeruddy-Thomas, E. Bukvareva, K. Davies, S. Demissew, G. Erpul, P. Failler, C. A. Guerra, C. L. Hewitt, H. Keune, S. Lindley, and Y. Shirayama. 2018. Assessing nature's contributions to people. Science 359(6373):270-272.

Di Pangracio, A., and N. A. Cáceres. 2020. Diagnóstico actualizado del estado de implementación Ley n 26331. 1a ed. Ciudad Autónoma Buenos Aires: Fundación Vida Silvestre Argentina; Fundación Ambiente y Recursos Naturales.

Dupleich, J. A., and G. Vicente. 2012. La agricultura familiar en el Dpto. La Paz. Pp. 37-49 en Experiencias de extensión actuando en los territorios. INTA EEA Paraná. Serie Extensión n 67.

Edwards, D. M., M. Jay, F. S. Jensen, B. Lucas, M. Marzano, C. Montagne, A. Peace, and G. Weiss. 2012. Public preferences across Europe for different forest stand types as sites for recreation. Ecology and Society 17:27. https: //doi.org/10.5751/ES-04520-170126.

Engler, P., M. Rodríguez, R. Cancio, M. Handloser, and L. Vera. 2008. Zonas agroeconómicas homogéneas de Entre Ríos. AEES INTA nº 6 .

FAO. 2016. El Estado de los bosques del mundo 2016. Los bosques y la agricultura: desafíos y oportunidades en relación con el uso de la tierra. Roma.

Ghazoul, J., Z. Burivalova, J. Garcia'Ulloa, and L. A. King. 2015. Conceptualizing forest degradation. Trends in Ecology and Evolution 30:622-632. https://doi.org/10.1016/j.tree.2015.08.001.

Guida Johnson, B., and G. Zuleta. 2013. Land-use land-cover change and ecosystem loss in the Espinal ecoregion, Argentina. Agriculture, Ecosystems and Environment 181:31-40. https://doi.org/10.1016/j.agee.2013.09.002. 
IPBES. 2018a. Summary for policymakers of the assessment report on land degradation and restoration of the Intergovernmental Science- Policy Platform on Biodiversity and Ecosystem Services. Scholes, R. J., L. Montanarella, E. Brainich, E. Brainich, N. Barger, B. Ten Brink, and L. Willemen (eds.). IPBES Secretariat. Bonn, Alemania. Pp. 44.

IPBES. 2018b. Summary for policymakers of the regional assessment report on biodiversity and ecosystem services for the Americas. Pp. 41 in J. Rice, C. S. Seixas, M. E. Zaccagnini, M. Bedoya-Gaitán, N. Valderrama, C. B. Anderson, M. T. K. Arroyo, M. Bustamante, J. Cavender-Bares, A. Díaz-de-Leon, S. Fennessy, J. R. García Márquez, K. García, E. H. Helmer, B. Herrera, B. Klatt, J. P. Olmeto, V. Rodríguez Osuna, F. R. Scarno, S. Schill and J. S. Farinaci (eds.). IPBES Secretariat. Bonn, Alemania.

Krieger, D. J. 2001. Economic Value of Forest Ecosystem Service: A Review. The Wilderness Society. Washington, D.C.

Laterra P., E. G. Jobbágy, and J. M. Paruelo (eds.). 2011. Valoración de servicios ecosistémicos: conceptos, herramientas y aplicaciones para el ordenamiento territorial. Buenos Aires: INTA.

López-Santiago, C., E. Oteros-rozas, B. Martín-López, T. Plieninger, E. Martín, and A. José. 2014. Using visual stimuli to explore the social perceptions of ecosystem services in cultural landscapes: the case of transhumance in Mediterranean Spain. Ecology and Society 19(2):27. https://doi.org/10.5751/ES-06401-190227.

MEA. 2005. Ecosystems and Human Well-being: Synthesis. Island Press, Washington, DC.

Maestre-Andrés, S., L. Calvet-Mir, and J. C. J. M. Van den Bergh. 2016. Sociocultural valuation of ecosystem services to improve protected area management: A multi-method approach applied to Catalonia, Spain. Regional Environmental Change 16:717-731. https://doi.org/10.1007/s10113-015-0784-3.

Martin-Lopez, B., I. Iniesta-Arandia, M. García-Llorente, I. Palomo, I. Casado Arzuaga, D. G. D. Amo, E. GómezBaggethun, E. Oteros-Rozas, I. Palacios-Agundez, B. Willaarts, J. A. González, F. Santos-Martin, M. Onaindia, C. López Santiago, and C. Montes. 2012. Uncovering ecosystem service bundles through social preferences. PLOS ONE 7:e38970. https://doi.org/10.1371/journal.pone.0038970.

Mastrangelo, M. E. 2018. Aproximaciones al estudio del comportamiento de los productores agropecuarios en el Chaco Seco. Ecología Austral 28:418-434. https://doi.org/10.25260/EA.18.28.2.0.644.

Mayring, P. 2014. Qualitative Content Analysis: Theoretical Foundation, Basic Procedures and Software Solution. Klagenfurt. https://doi.org/10.1007/978-94-017-9181-6_13.

MEA 2005. Ecosystems and human well-being. Millennium Ecosystem Assessment. Island Press, Washington, D.C., USA. Press, Washington, D.C. USA.

Menzel, S., and J. Teng. 2010. Ecosystem services as a stakeholders driven concept for conservation science. Conservation Biology 24(3):907-909. https://doi.org/10.1111/j.1523-1739.2009.01347.x.

Mendizábal, N. 2006. Los componentes del diseño flexible en la investigación cualitativa. En V. de Gialdino (ed.). Estrategias de investigación cualitativa. Biblioteca de Educación. Editorial Gedisa. ISBN: 978-84-9784-173-3.

Ministerio de Ambiente y Desarrollo Sustentable (MAyDS). 2016. Monitoreo de la superficie de bosque nativo de la República Argentina. Unidad de Manejo del Sistema de Evaluación Forestal.

Ministerio de Ambiente y Desarrollo Sustentable (MAyDS). 2017. Monitoreo de la superficie de bosque nativo de la República Argentina. Unidad de Manejo del Sistema de Evaluación Forestal.

Muñoz, J. D., S. Milera, C. Romero, and A. B. Brizuela. 2005. Bosques nativos y selvas ribereñas en la Provincia de Entre Ríos. Pp. 550 en F. G. Aceñolaza (eds.). Temas de la Biodiversidad del Litoral Fluvial Argentino II. Serie INSUGEO: Miscelánea 14.

Nanni, A. S, M. Piquer-Rodríguez, D. Rodríguez, M. Nuñez-Regueiro, M. E. Periago, S. Aguiar S. Ballari, C. Blundo, E. Derlindati, Y. Di Blanco, A. Eljall, R. H. Grau, L. Herrera, A. Huertas Herrera, A. E. Izquierdo, J. Lescano, L. Macchi, F. Mazzini, M. Milkovic, L. Montti, A. Paviolo, M. Pereyra, R. D. Quintana, V. Quiroga, D. Renison, M. Santos Beade, A. Schaaf, and N. I. Gasparri. 2020. Presiones sobre la conservación asociadas al uso de la tierra en las ecorregiones terrestres de la Argentina. Ecología Austral 30:304-320. https://doi.org/10.25260/EA.20.30.2.0.1056.

Ninan, K. N., and A. Kontoleon. 2016. Valuing forest ecosystem services and disservices - Case study of a protected area in India. Ecosyst Serv 20:1-14. https://doi.org/10.1016/j.ecoser.2016.05.001.

Quétier, F., E. Tapella, G. Conti, D. M. Cáceres, and S. Díaz. 2007. Servicios ecosistémicos y actores sociales. Aspectos conceptuales y metodológicos para un estudio interdisciplinario. Gaceta ecológica 84-85:17-27.

Rojas Crotte, I. R. 2011. Elementos para el diseño de técnicas de investigación: una propuesta de definiciones y procedimientos en la investigación científica. Tiempo de Educar 12(24):227-297.

Sabattini, R. A., M. G. Wilson, N. Muzzachiodi, and A. F. Dorsch. 1999. Guía para la caracterización de los agroecosistemas del centro-norte de Entre Ríos. Revista Científica Agropecuaria 3:7-19.

Sabattini, R. A. 2009a Informe 3. Zonificación de los bosques nativos en el Departamento Feliciano según las categorías de conservación. FCA UNER y la Dirección General de Recursos Naturales de la Secretaria de la Producción del Gobierno de Entre Ríos. Pp. 20.

Sabattini, R. A. 2009b. Informe 1. Zonificación de los bosques nativos en el Departamento La Paz según las categorías de conservación. FCA UNER y la Dirección General de Recursos Naturales de la Secretaria de la Producción del Gobierno de Entre Ríos. Pp. 20.

Sautu, R., P. Boniolo, P. Dalle, and R. Elbert 2005. La construcción del marco teórico en la investigación social. En R., Sautu et al. (eds.). Manual de metodología. Construcción del marco teórico, formulación de los objetivos y elección de la metodología. ISBN 987-1183-32-1.

Schmidt, M. 2018. Una década protegiendo los bosques nativos. Claroscuros de una política ambiental en defensa de los bienes comunes. Revista Bordes. Universidad Nacional de José C. Paz. Buenos Aires. 
Scholte, S. S. K., A. J. A. van Teeffelen, and P. H. Verburg. 2015. Integrating socio-cultural perspectives into ecosystem service valuation: a review of concepts and methods. Ecological Economics 114:67-78. https://doi.org/10.1016/ j.ecolecon.2015.03.007.

Secretaría de Ambiente y Desarrollo Sustentable (SAyDS). 2007. Primer Inventario Nacional de Bosques Nativos. Segunda etapa. Inventario de campo de la región del Espinal. Distritos de Caldén y Nandubay. Proyecto Bosques Nativos y Áreas Protegidas, Préstamo BIRF 4085-AR.

Simula, M. 2009. Towards Defining Forest Degradation: Comparative Analysis of Existing Definitions. FAO. Forest Resources Assessment Programme, Working Paper 154. Pp. 59.

Sione, S., R. A. Sabattini, S. Ledesma, F. Dorsch, and C. Fortini. 2006. Caracterización florística y estructural del estrato arbustivo de un monte en pastoreo. Revista Científica Agropecuaria 10:59-67.

Soneira, A. J. 2006. La Teoría Fundamentada en los datos (Grounded Theory) de Glaser y Strauss. En V. de Gialdino (ed.). Estrategias de investigación cualitativa. Biblioteca de Educación. Editorial Gedisa. ISBN: 978-84-9784-173-3.

Spahn, E. 2013. Modelo de estados y transiciones para los bosques y pastizales del norte entrerriano. Tesis de Maestría. Universidad Nacional de Río Cuarto. Córdoba, Argentina. Pp. 184.

Sutrop, U. 2001. List task and a cognitive salience index. Field Methods 13(3):263-276. https://doi.org/10.1177/ $1525822 \times 0101300303$.

Tapella, E. 2012. El Conflicto Social en Torno a la Apropiación de Servicios Ecosistémicos en el Oeste de la Provincia de Córdoba. La Posición de los Actores Sociales más Vulnerables. Tesis doctoral, Universidad Nacional de Córdoba, Argentina.

Tasi, H., M. Wilson, G. Schulz, N. Indelángelo, and D. Bedendo. 2007. Uso de la tierra en el área de bosques nativos de Entre Ríos. Pp. 33-46 en O. P. Caviglia, O. F. Paparotti and M. C. Sasal (eds.). Agricultura Sustentable en Entre Ríos. Ediciones INTA.

Vang Rasmussen, L., C. Watkins, and A. Agrawal. 2017. Forest contributions to livelihoods in changing agricultureforest landscapes. Forest Policy and Economics 84:1-8. https://doi.org/10.1016/j.forpol.2017.04.010.

Wilson, M., and R. Sabattini. 2001. Sustentabilidad de los agroecosistemas de montes de Entre Ríos: revisión crítica y modelos conceptuales. Revista Facultad de Agronomía 21(2):117-128. 